\title{
L'illocutoire du dispositif (How not to do nothing with links)
}

The Illocutionary Force of Tools: How Not to Do Nothing with Links

Jean-Thierry Julia

\section{CpenEdition}

Journals

Édition électronique

URL : http://journals.openedition.org/edc/383

DOI : $10.4000 /$ edc.383

ISSN : 2101-0366

Éditeur

Université Lille-3

Édition imprimée

Date de publication : 1 décembre 2006

Pagination : 119-130

ISBN : 2-9514961-7-6

ISSN : $1270-6841$

Référence électronique

Jean-Thierry Julia, «L'illocutoire du dispositif (How not to do nothing with links) », Études de

communication [En ligne], 29 | 2006, mis en ligne le 20 novembre 2014, consulté le 30 avril 2019. URL: http://journals.openedition.org/edc/383 ; DOI : 10.4000/edc.383

Ce document a été généré automatiquement le 30 avril 2019.

(c) Tous droits réservés 


\title{
L'illocutoire du dispositif (How not to do nothing with links)
}

The Illocutionary Force of Tools: How Not to Do Nothing with Links

\author{
Jean-Thierry Julia
}

1 Dès 1960, Joseph C. R. Licklider, psycho-acousticien rompu aux techniques informatiques naissantes, aura l'intuition, au lieu d'un calculateur central et des informaticiensprogrammeurs qui s'y affairent, d'une « interaction » possible, directe, entre l'ordinateur et son utilisateur humain: dans un article fondateur, il entrevoit le calculateur « interactif» ( «interactive computer », Licklider, 1960), et sera l'initiateur d'un vocable qui connaitra par la suite le succès que l'on sait. Entre temps, et notamment à la suite de Michel Foucault, l'objet technique sera devenu « dispositif » socio-technique : « [...] du dit, aussi bien que du non-dit, voilà les éléments du dispositif» (Foucault, 1977). Ce qu'il est, et ce qu'on en fait - l'interactivité y aidera ; ce qui est dit, et ce qui n'est pas dit...

2 Ce qu'encore il est dit, sans pour autant être dit, auraient pu ajouter John L. Austin ou John R. Searle : c'est cette veine du «in saying» interactif que nous voudrions aborder dans cet article. Et viser, de façon peut-être originale au sein des sciences de l'information et de la communication, à investir plus profondément ce type d'« échange ", noué au creux de l'« interaction » entre l'utilisateur humain et le dispositif technique interactif, dyade dès lors objet de toutes les réflexions en la matière.

3 Renvoyant pour plus de précision à d'autres de nos études (Julia, 2003), nous entendrons dans ce qui suit par « dispositif interactif», le " dispositif » au sens strictement foucaldien du terme, comme suggéré ci-dessus, et notamment, ce qui sera conforme plus loin à nos analyses, comme "[...] stratégies de rapports de forces supportant des types de savoir, et supportés par eux» (Foucault, 1977); ce dispositif sera dit " interactif » quand il sera susceptible d'être le siège - ou mieux, le lieu de la représentation (Boissier, 2000) - d'une interaction avec son utilisateur humain. 


\section{Énonciation interactive et modélisation conversationnelle}

4 En matière de consultation de multimédias interactifs ${ }^{1}$, les réflexions autour de la notion d'interactivité ont ainsi amené à étudier les modalités de production de ces «énoncés " multimédias (Mabillot, 2000; Julia, 2003). Sous l'angle énonciatif, l'interaction entre l'utilisateur et le dispositif multimédia - ou plutôt, quoiqu'alors absent, l'auteur du produit multimédia - s'entrevoit comme la possibilité pour le premier d'assumer activement auprès $d u$ second, une part du processus d'énonciation, c'est-à-dire du processus de réalisation effective - nous préfèrerons d'«instanciation »- de l'énoncé multimédia. Quand bien même il n'en sera pas créateur, l'utilisateur contribuera de la sorte et tour à tour à une telle instanciation des énoncés. Nous pourrions alors avancer qu'au creux des dispositifs interactifs, de tels énoncés, non encore instanciés, demeurent néanmoins latents. En amont de ces étapes liées à leur instanciation par l'utilisateur, et ainsi dès leur conception par l'auteur du produit multimédia, de tels "pré-énoncés " s'insèrent néanmoins dans une "structure discursive, [jusque-là] incomplète " (Mabillot, $2000,49)$.

De façon renouvelée au fil d'une telle session interactive de consultation, l'utilisateur viendra compléter par ses propres contributions et faire dérouler le discours multimédia. Aussi, le dispositif dans son ensemble aura tôt fait de recréer l'illusion de l'échange conversationnel - au fondement de la communication. Nous verrons que ce ne sera le constat que d'un instant...

6 La problématique évoquée est corollaire de celle que soulèvent encore Emmanuël Souchier, Yves Jeanneret et Joëlle Le Marec à travers la notion de « signe passeur », signes qui « n'assurent pas seulement une fonction instrumentale permettant de 'circuler' dans le texte lui-même, mais présentent également une fonction sémiotique complexe [...]» (Souchier et al., 2003, 23), confirmant par là dans le champ des SIC un objet incontournable pour aborder l'interactivité. Mais les auteurs plus loin de constater que «la complexité de ces signes passeurs, unis par une même contrainte logicielle mais interprétés, organisés et énoncés différemment demanderait un travail de qualification sémiopragmatique ", et de surseoir momentanément à une telle étude; ce sera précisément à une telle approche, sémiopragmatique, que s'attachera la suite de notre réflexion.

7 Dans le prolongement des réflexions philosophiques pragmatiques inaugurées par Austin (1962, trad. fr. 1970), approfondies par Searle (1969, trad. fr. 1972), certaines recherches linguistiques, relatives dès lors à l'analyse des discours en situation, se seront en effet attachées à de telles modélisations, plus particulièrement à celle de la conversation. Les travaux de l'École de Genève, entre autres, dans une perspective cognitiviste interactionniste autour d'Eddy Roulet ou de Jacques Moeschler, aboutissent à l'élaboration d'un modèle structurel (Roulet et. al., 1985), auquel nous voudrions emprunter certains résultats tant ils paraissent pertinents dans le cadre de nos investigations : bien qu'il ne relève certes pas de la stricte conversation, nous étudierons, dans la situation de consultation interactive, comment un tel "échange ", entendu ici comme alternance de contributions de l'utilisateur et d'autres de la machine, pourra être caractérisée au vu de telles notions. 
8 Le modèle genevois s'attache à la dimension hiérarchique de l'échange conversationnel, autour de trois unités discursives que seront l'acte, l'intervention et l'échange :

- l'acte, ou « acte textuel » (et non plus « acte de langage », quand la notion chez Roulet [2001, 63] se sera progressivement éloignée de celle de Searle) est l'unité minimale de l'énonciation: il est le fait d'un seul interlocuteur (caractère monologal), encore marqué d'une unique contribution argumentative au discours (caractère monologique), dont quelques exemples pourraient être « Paul est là ? », « Paul est parti! », « Il est six heures... », ou bien encore « Déjà ? !... »;

- l'intervention, monologale, ou dialogale par imbrication récursive de constituants, pourra consister en un seul acte (intervention monologique), ou articuler à des fins argumentatives le constituant "principal» d'un acte ou d'une intervention et des constituants «subordonnés", préalables ou postérieurs, qui pourront être des actes ( $J e$ cherche Paul, il est là?») ou récursivement, pour une intervention éventuellement dialogique, des interventions ou des échanges («Salut Virginie. Je cherche Paul, apparemment il est pas là... Euh... T'as vu l'heure ?... - Oui, je pensais qu'il serait encore là... Ciao !») ;

- l'échange consiste quant à lui en de seules interventions des protagonistes: deux interventions pour l'échange d'ouverture ou de clôture («Bonjour Virginie! - Bonjour... », « $A u$ revoir, Paul... - Ciao!»); deux encore pour l'échange "confirmatif» («Il fait beau aujourd'hui! - Oui, c'est l'été!»), trois pour l'échange " réparateur $»^{2}$, notamment évoqué par Erwin Goffman (1974) ; voire cinq, sept ou davantage, dans le cas de réactions négatives qui ne permettraient pas alors de clore plus tôt l'échange. La transaction conversationnelle globale pourra quant à elle être constituée de plusieurs échanges successifs.

9 Concernant dès lors le dispositif interactif, il sera possible de discerner dans l'interaction qui s'y joue, de semblables unités discursives, ici de façon dissymétrique. Pour l'instant autour d'une banale "navigation », consultation de pages et documents au fil de liens hypertextes, nous conviendrons qu'une structure homologue à l'échange puisse s'observer dans l'alternance des contributions à l'énoncé multimédia: instanciation de textes, images et sons, du côté du dispositif technique ; pointage d'un item et clic, du côté de l'utilisateur. Si l'intervention côté dispositif se révèle assurément composite et complexe - gardons encore qu'elle puisse receler quelque récursivité -, celle de l'utilisateur s'en verra d'autant simplifiée, en un ou deux « actes » élémentaires (clic, ou pointage puis clic) - selon une acception de l'acte, ici non-verbal, qu'il faudrait encore affiner -, peut-être plus encore quand il s'agira préalablement de pouvoir qualifier et interprêter un tel "signe passeur » dans toute sa complexité sémiotique (Souchier et al., 2003).

\section{L'illocutoire conversationnel}

10 De façon complémentaire à cette description hiérarchique, le modèle genevois s'attache encore à une analyse "fonctionnelle » (Roulet et. al., 1985), rebaptisée " relationnelle » (Roulet et. al., 2001), visant à identifier les relations entre constituants. Les relations « interactives" seront tout d'abord mises au jour entre les différents constituants de l'intervention, et ont trait à leur articulation dans un registre argumentatif ${ }^{3}$. L'analyse relationnelle souligne en outre, pour chaque intervention dans l'échange - ce qui nous intéressera ici plus particulièrement au vu de notre objet -, une fonction ou relation illocutoire, au sens premier des réflexions performatives d'Austin ou Searle, c'est-à-dire non-constitutive de l'énoncé lui-même (locutoire), mais néanmoins sous-jacente, inscrite 
au creux de cet énoncé (illocutoire). Cette fonction n'en sera pas moins effective, au-delà de l'énoncé jusque-là déroulé, avec quelque conséquence, verbale ou non, du côté de l'un ou de l'autre des interlocuteurs (perlocutoire). Roulet confirme une telle «force illocutoire » attachée aux interventions constitutives de l'échange, pour l'essentiel sous deux occurrences: d'une part fonction illocutoire initiative d'une intervention, qui donne des droits ou impose des contraintes à l'autre partenaire (assertion, demandes d'information [question], de confirmation, requête, offre, etc.); d'autre part fonction illocutoire réactive d'une intervention qui vient se conformer (Roulet dira «favorable») en retour à l'illocutoire de l'intervention initiative précédente - que cette réaction soit positive ou négative (resp. ratification/évaluation négative, réponse positive/négative, confirmation/infirmation, exécution/refus, acceptation/rejet, etc. [d'après Moeschler, 1985]). L'intervention qui ouvre l'échange a force d'initiative, les suivantes peuvent conjuguer une double relation illocutoire, réactive à la précédente et initiative de la suivante, enfin l'intervention qui vient clore un échange présente avec la précédente une relation strictement réactive - en général d'évaluation positive ou de ratification - et sera dite évaluative.

Les fonctions illocutoires des interventions qui constituent l'échange permettent alors d'en vérifier la complétude interactionnelle (ou dialogique): "Si [la réaction de l'interlocuteur] est favorable, le locuteur peut clore la négociation en exprimant à son tour son accord [...]. Nous qualifierons de complétude interactionnelle la satisfaction de cette contrainte du double accord qui autorise la clôture d'une négociation " (Roulet et. al., 1985). Si la réaction de l'interlocuteur est défavorable, c'est-à-dire ne satisfait pas la fonction illocutoire de l'intervention précédente (indépendamment encore de son caractère positif ou négatif), la condition de complétude interactionnelle n'est pas remplie, le locuteur ne peut clore l'échange et poursuit l'interaction jusqu'à aboutir à un autre accord: "Il est où, Paul ? - Quiça ? - Paul! - Ah, Paul! Oui, il est là... - Merci », ou indifféremment "[...] Euh, non, y'a pas de Paul ici... - Merci quand même ». La complétude interactionnelle de l'échange, fût-il confirmatif, réparateur ou autre, repose bien dans tous les cas sur un tel double accord, où la dernière intervention évaluative est encore conforme à l'illocutoire de la précédente, et clôt éventuellement l'échange : «Tu n'as pas vu Paul ? - Non, pas récemment ... », ou « Tu n'as pas vu Paul ? - Il est malade, Paul... - Ah bon... », ou bien encore « Tu n'as pas vu Paul ? - Qui ça ? - Paul !... - Oui, il est là... ».

\section{L'illocutoire réactif}

12 Sous couvert de telles notions complémentaires, nous pourrions d'ores et déjà entrevoir que le «dialogue » mis en œuvre autour du dispositif interactif ne puisse s'avérer, pour l'interlocuteur humain, qu'un figement dans la position réactive : les énonciations côté dispositif (initiatives à mettre au compte de l'auteur du produit interactif) pourront ainsi être considérées comme autant de sollicitations - illocutoires - envers l'utilisateur, qui dicteront à celui-ci en retour, si ce n'est l'item choisi, tout du moins l'assignation à ne pouvoir qu'effectuer un tel choix. Et conviendra-t-on pour l'instant avec Françoise Séguy (1999, 74), qu'en place d'interactivité, nous n'avions jusqu'ici affaire qu'à simple réactivité.

Remarquons sur ce point que si l'intervention de l'utilisateur (choix puis clic) a pu un temps paraitre à quelques thuriféraires technophiles comme initiative d'une requête enjoignant la réalisation du prochain énoncé - ce à quoi s'exécute le dispositif -, cela n’aura été que par méprise, à l'image de celle que soulignait Fillmore (1973), quand il 
remarquait que l'on permettait souvent aux visiteurs sollicitant l'entrée dans la pièce en leur ordonnant de le faire («Entrez!») $\ldots$ Lira-t-on alors dans la page-écran multimédia, derrière chaque lien, quelque proposition - intrusive ? - de développer plus avant ce qui est pour l'instant instancié ; et plutôt que d'y reconnaître, si ce n'est l'intrusion, tout du moins l'initiative (d'un auteur absent), l'utilisateur préférera s'en penser l'ordonnateur, et, afin de sauver la face (humaine) devant le dispositif technique, aura tôt fait d'y lire un renversement de l'initiative en sa faveur...

Notons encore que derrière la notion de «signe passeur " évoquée plus haut, et les constats qu'elle suscite (« ensemble des signes présents sur l'écran », «Et alors là, où je clique? ", [Souchier et al., 2003, 101; 117]) les auteurs puissent alors ne privilégier qu'une approche réactive (l'auteur - l'autre? - étant encore, sous couvert d'une qualification prêtant à personnification du signe, le "pilote " explicitement attendu); signe qu'en outre, nous préférerions désigner plus simplement par le terme de "connecteur ", déjà utilisé dans le champ ${ }^{5}$ et ne renvoyant alors qu'au texte ou au discours - fût-il multimédia - , et dès lors à ce que l'on en fait.

Aussi, la transaction interactive ne semble pas pleinement coïncider, dans l'ordre des actes « exercitifs » d'Austin ou «directifs » de Searle, ni avec l'alternance autour d'une question, à laquelle se résoudrait l'utilisateur par la réponse de son clic, ni avec celle autour d'une requête - fût-elle en matière langagière (Kerbrat-Orecchioni, 2001, 85) -, dont ce même utilisateur prendrait l'initiative dans l'injonction d'un clic au dispositif. Nous évoquerons une autre figure énonciative, notamment plus conforme à la configuration illocutoire observée. Il s'agit de la figure de l'offre - ici d'une offre en contenus informationnels -, qui, sous couvert d'une proposition du côté du dispositif, cumule non seulement le tour « directif » de la question/réponse (amenant l'utilisateur à «dire» son acceptation ou son rejet), mais encore celui "promissif» de l'accomplissement prochain, conforme à la proposition faite (amenant le dispositif initiateur à faire, ou en l'occurrence à «dire » en instanciant de prochains contenus multimédias). Ici l'offre « promissive » consiste, après l'acceptation que constitue le choix de l'utilisateur, que soit effectivement tenue la promesse de délivrer de tels contenus. Ainsi, si la contribution finale du dispositif a pu paraître un temps « réactive », ce n'est pas tant en réponse à quelque initiative d'un clic d'injonction de l'utilisateur, mais bien de façon conséquente à l'initiative du côté du dispositif lui-même : engagement illocutoire, inscrit (par l'auteur) au creux de l'offre, de se conformer par la suite à la proposition faite, et in fine - effet perlocutoire - d'instancier les contenus relatifs au choix effectué.

Et nous pourrions momentanément conclure à des contributions dissymétriques, interventions « réactives » relevées non seulement du côté de l'utilisateur (acceptation par son choix), mais aussi du côté du dispositif (instanciation suivante), et en revanche, interventions initiatives (offre de prochains contenus) du seul côté du dispositif. Si dans de telles configurations interactives l'utilisateur n'est finalement pas le seul à être assujetti à une position réactive, nous confirmons cependant, à ce point de l'analyse, qu'il ne se retrouve jamais en position initiative...

\section{L'illocutoire interactif}

17 Dans le schéma exposé plus haut, si ce n'est rejeter et se détourner de l'offre faite en y mettant un terme (interrompre l'échange et/ou fermer l'outil de consultation), il est impossible pour l'utilisateur sollicité de ne pas se conformer, monologiquement, au fait 
même de l'offre : la clause de complétude interactionnelle s'en trouve ainsi éminemment satisfaite, et ce au terme de chaque tour d'interventions dispositif/utilisateur... Mais convenons qu'il s'agirait bien là d'un piètre échange, monologique et proche du discours «béni-oui-oui » d'un utilisateur-godillot, symptomatique des dispositifs "pressebouton » : offre-acceptation-instanciation, offre-acceptation-instanciation, ad libitum.

Toutefois, à l'occasion d'« échanges » avec un dispositif, pourrions-nous évoquer quelques « incomplétudes interactionnelles » - provisoires - ou d'autres figures qui pourront venir densifier et prolonger le tour d'interaction dans le sens de transactions plus complexes. Et l'utilisateur dès lors de ne plus être assigné à une position réactive, et de retrouver quelque initiative au sein de l'échange. De telles configurations sont attestées à l'occasion d'autres situations interactives que ne saurait résumer seule la navigation "réactive " (offre-acceptation) envisagée jusqu'ici. L'exemple canonique de telles figures réside dans l'initiative - salvatrice - de l'utilisateur (" pomme+alt+esc » sur Mac ou «Ctrl+Alt+Suppr » sur PC), qui, en contexte dysfonctionnel du dispositif («bouclant» par ex. autour d'une perpétuelle requête réactive erronée) permet de forcer à quitter l'application défectueuse... Dans le cas d'un moteur de recherche - et à l'inverse d'un annuaire, tout réactif -, il s'agit temporairement de ne pas clore le tour d'interaction et de repousser la complétude interactionnelle après l'inscription textuelle de sa requête dans le champ prévu à cet effet ("Venise », ou même encore, avec corrections «Ital Venise »); avant de compléter et terminer enfin l'échange en cliquant sur le bouton « Ok » ou « rechercher » (le service propose alors les résultats correspondants à la requête, sous forme de liens vers les pages en question; contexte à nouveau totalement réactif).

Nous avons pu évoquer (Julia, Lambert, 2003), à l'exact opposé de stratégies réactives, les configurations historiques informatiques autour de langages en lignes de commande (par ex. du type « copy c:/jtj/etudes_de_comm/article.doc d: »). Le cas d'école est intéressant dans le sens où l'utilisateur a bien ici entière initiative d'une requête envers le dispositif sous couvert néanmoins d'apprentissage préalable et de maîtrise dudit langage de commande. Soulignons alors que c'est de vouloir dégager l'utilisateur de cette (lourde) part d'initiative, décontenancé qu'il était devant de tels langages abscons, que les interfaces graphiques tireront leur succès; de tels dispositifs consisteront dès lors à ne présenter que de seules figures réactives ${ }^{6} \ldots$

Il s'agira ici pour nous d'entrevoir, ni le tout initiatif du langage de commande, ni le tout réactif de l'offre graphique, d'autres configurations intermédiaires de «juste » activité pour l'utilisateur, entre épreuve initiative et facilitation réactive. Ce sont en effet autour de telles configurations que se retrouvent, à des degrés divers, certaines catégories de dispositifs interactifs (de façon non exhaustive, éducatifs, culturels, ou bien encore ludiques, parfois commerciaux), fondés sur une co-construction, ici plus conséquente que l'acceptation, dans l'énonciation de prochains contenus. Que ce soit en ré-agençant les items relatifs à une notion, en explorant une partie d'un musée au fil d'une "visite virtuelle ", ou en se démenant dans l'univers foisonnant d'un jeu, le dispositif convoque alors une part d'initiative de l'utilisateur, et se dérobe à fournir de façon exhaustive une liste de l'ensemble des possibles, parmi lesquels comme dans l'offre auctoriale, il conviendrait uniquement de choisir: didacticiel d'astronomie, où il s'agit d'initier le mouvement et de faire glisser la lune sur son orbite afin de visualiser l'éclipse ; site web de l'abbaye de Conques, où l'immersion totale dans l'image ne renvoie qu'implicitement au mouvement de la souris pour " parcourir » l'espace ; site artistique d'ez3kiel où il s'agit d'ores et déjà d'explorer l'interface laconique de la page d'accueil ; enfin sites d'Agnès B., 
Havaianas, Issey Miyake, Adidas ou bien encore Absolut, où il convient d'être à l'initiative de mouvements du pointeur par le biais de la souris, pour enclencher textes et ambiance musicale, animation graphique, diaporama photo, vidéos, ou bien encore quelque jeu... Notons encore, points d'orgue de la figure, que les simulateurs de tout ordre renvoient éminemment à de telles modalités interactives, tour à tour initiatives et réactives, que nous pourrions alors qualifier de « prospectives ».

Enfin, avec Jean-Louis Boissier qui l'illustre magistralement dans sa lecture personnelle de l'œuvre de Jean-Jacques Rousseau (2000) et ses saynètes de vidéo interactive que l'on «manipule» de façon inédite, de conclure autour de figures illocutoires affûtées de la sorte au fil de l'« échange » avec le dispositif : "L'interactivité n'est-elle pas ce qui simule des interactions réelles?» (id., 11) ; mais bien alors tout type d'interactions, fussent-elles, de la part de l'utilisateur, réactives tout aussi bien qu'initiatives.

\section{BIBLIOGRAPHIE}

Austin, J. L., (1962), How to do Things with Words, Oxford, Oxford University Press, trad. fr. 1970, Quand dire c'est faire, Paris, Le Seuil.

Boissier, J.-L., (2000), « Le moment interactif », Moments de Jean-Jacques Rousseau. Confessions et Rêveries, Paris, Gallimard multimédia, 2000, CD-Rom (livret). Communications, (1980), n³2, Les actes de discours.

Foucault, M., (1977), « Le jeu de Michel Foucault » (entretien), Ornicar ? Bulletin périodique du champ freudien, $n^{\circ} 10$, pp. 62-93, rééd. 2001, in : Dits et écrits II (1976-1988), Paris, Gallimard, coll. Quatro, pp. 298-329.

Goffman, E., (1967), Ritual Interaction. Essays on Face-to-face Behavior, New York, Doubleday, trad. fr. 1974, Les rites d'interaction, Paris, Éditions de Minuit.

Fillmore, C. J., (1973), « May We Come ln? », Semiotica, n 9, pp. 97-115.

Julia, J.-T., (2003), « Interactivité, modes d'emploi. Réflexions préliminaires à la notion de document interactif », Documentaliste-Sciences de l'information, vol. 40, n 3, juin, pp. 204-212.

Julia, J.-T. et Lambert, E., (2003), « Énonciation et interactivité : du réactif au créatif », Communication \& Langages, $\mathrm{n}^{\circ} 137$, in : Després-Lonnet M. (dir.), Interactivité : attentes, usages et socialisation, dossier, octobre, pp. 30-44.

Kerbrat-Orecchioni, C., (2001), Les actes de langage dans le discours. Théorie et fonctionnement, Paris, Nathan, coll. Fac., série « linguistique ».

Licklider, J. C. R., (1960), « Man-Computer Symbiosis », IRE (actuel. IEEE), vol. HFE-1, Transactions on Human Factors in Electronics, March, pp. 4-11.

Mabillot, V., (2000), Mises en scènes de l'interactivité. Représentations des utilisateurs dans les dispositifs de médiations interactives, thèse de doctorat en Sciences de l'information et de la communication, Université Lyon 2. 
Moeschler, J., (1985), Argumentation et conversation, éléments pour une analyse pragmatique du discours, Paris, Hatier.

Roulet, E., (1980), « Modalité et illocution. Pouvoir et devoir dans les actes de permission et de requête », Communications, $\mathrm{n}^{\circ}$ 32, Les actes de discours, pp. 216-239.

Roulet, E., (1999), La description de l'organisation du discours. Du dialogue au texte, Paris, Didier, coll. Langues et apprentissage des langues.

Roulet, E., Auchlin, A., Moeschler, J., Rubattel, C. et Schelling, M., (1985), L'articu-lation du discours en français contemporain, Berne, Peter Lang.

Roulet, E., Filliettaz, L., Grobet, A., Colla, B. et Burger, M., (2001), Un modèle et un instrument d'analyse de l'organisation du discours, Berne, Peter Lang, coll. Sciences pour la communication, $\mathrm{n}$ ${ }^{\circ} 62$.

Searle, J. R., (1969), Speech Acts, Cambridge, Cambridge University Press, trad. fr. 1972, Les actes de langage, Paris, Hermann.

Séguy, F., (1999), Les produits interactifs et multimédias. Méthodologies, conception, écritures, Grenoble, Presses universitaires de Grenoble, coll. « La communication en plus ».

Souchier, E., Jeanneret, Y. et Le Marec, J., (dir.), (2003), Lire, écrire, récrire. Objets, signes et pratiques des médias informatisés, Paris, BPI/Centre Pompidou, coll. «Études et recherche».

\section{NOTES}

1. Indifféremment en ligne ou hors ligne, qu'ils soient sites web, CD-ROM, borne interactive, etc., élaborés dans un quelconque format, HTML, XML, Flash, Director, etc., ou même encore format de pré-AO (Impress, PowerPoint...). Les courriels, forums, chats et autres « clavardages », selon nous « interactionnels » et non interactifs, ne sont pas ici objet de notre réflexion.

2. L'intervention d'un premier protagoniste pourra en effet être vécue par l'autre comme une intrusion dans son espace personnel, qu'il conviendra de « réparer » pour retrouver l'équilibre rituel social («Allo ! Bonjour. Euh... Excusez-moi. Je voudrais parler à Virginie, est-ce qu'elle est là ? - Oui, la voilà, je vous la passe... - Merci... »).

3. Les relations interactives sont selon l'auteur au nombre de huit : argument, contre-argument, reformulation, topicalisation, succession, préalable, commentaire, clarification (Roulet et. al., 2001, 172).

4. "Sachant que l'interlocuteur peut avoir l'impression, quand il entre dans la pièce où nous nous trouvons, qu'il est un intrus [...] le meilleur moyen de faire comprendre que cette entrée n'est pas indésirable [...] c'est d'exprimer que nous y tenons beaucoup [...] en ordonnant à l'interlocuteur d'entrer » (Roulet, 1980, 237).

5. Connecteur non plus syntaxique ou sémantique, comme habituellement en sciences du langage, mais pragmatique, voire rhétorique, c'est-à-dire lié à l'organisation du discours.

6. Ce que nous pourrions rapprocher - mais qui se situerait trop loin de notre objet - des velléités humaines soulignées par Stanley Milgram à se complaire en situation de soumission à autorité (ici de l'auteur); ou encore, dans le sens de Jürgen Habermas, d'une rationalité instrumentale (le travail) au détriment d'un agir communicationnel (l'interaction)... 


\section{RÉSUMÉS}

Qu'en est-il tout d'abord de la notion d'interactivité ? Quand cette dernière renverra à la notion d'interaction et à celle d'énoncés multimédias, le texte interroge ensuite les spécificités d'une telle interaction discursive. L'échange avec le dispositif interactif sera analysé sur la base de résultats issus des théories linguistiques de l'énonciation, notamment à travers le modèle du discours conversationnel élaboré au sein de l'École de Genève par Eddy Roulet. Le modèle permettra ainsi de dégager la dimension illocutoire des interventions de chacun des interlocuteurs, ici l'utilisateur et le dispositif technique, et les relations initiative ou réactive de ces interventions. Nous montrerons alors que l'utilisateur est le plus souvent astreint à une position réactive - dans la transposition sur dispositif d'une figure de l'offre; toutefois, d'autres configurations interactives, autrement finalisées, permettront à l'utilisateur de retrouver toute sa part d'initiative...

What is interactivity all about? Because the notion of interactivity is linked to the notion of interaction just as it is with multimedia communication, we should ask what kind of discursive interaction this implies. To answer this question, the exchanges within the interactive mechanism are analyzed here according to the linguistic theory of enunciation and the modeling of conversation by Eddy Roulet and the Geneva School. This theoretical framework suggests that illocutionary force lies behind both the moves of the computer and those of the user, that is, a phenomenon that could be defined as a set of either proactive or reactive relations. However, our study shows that most of the time, the position of the user can be seen as reactive in relation to the interactive nature of the offer, even if in some cases of interaction, the initiative can be handed over to the user.

\section{INDEX}

Mots-clés : interactivité, École de Genève, communication multimédia

Keywords : interactivity, École de Genève, multimedia communication

\section{AUTEUR}

\section{JEAN-THIERRY JULIA}

Jean-Thierry Julia, de par une double formation universitaire en sciences humaines et sociales [ sic] et sciences exactes (informatique linguistique), Jean-Thierry Julia s'attache dans ses travaux de recherche à des approches croisées - communicationnelle, pragmatique et informatique - des dispositifs techniques de communication : notion d'interactivité - sous l'angle des théories de l'énonciation -, écriture et scénarisation multimédia, NTIC et espace public, ou encore théorie algorithmique de l'information. Adresse électronique : jean-thierry.julia@iut-tlse3.fr. 